\title{
La creencia en fake news y su rol en el acatamiento de medidas contra COVID-19 en México
}

\author{
The role of believing fake news on compliance \\ of anti-COVID-19 measures in Mexico
}

\author{
Rocío Galarza-Molina \\ Universidad Autónoma de Nuevo León, México \\ rociogalarzamolina@gmail.com \\ https://orcid.org/0000-0001-7002-0638 \\ Carlos Muñiz \\ Universidad Autónoma de Nuevo León, México \\ carlos.munizm@uanl.mx \\ https://orcid.org/0000-0002-9021-8198
}

Recibido: 13/11/2020 Revisado: 19/03/2021 Aceptado: 16/04/2021 Publicado: 01/09/2021

\begin{abstract}
Resumen
Durante la pandemia por la COVID-19, las fake news proliferaron y con ello la preocupación de que estas afecten el comportamiento ante la enfermedad. Mediante una encuesta semi-representativa en México $(\mathrm{N}=1211)$, este estudio analiza un proceso mediacional para determinar el impacto del uso de medios tradicionales y sociales en el acatamiento de medidas de prevención de contagio, a través de la percepción de veracidad de fake news sobre COVID-19. Como se anticipaba, los resultados indican que creer en noticias falsas conduce a un menor cumplimiento de medidas preventivas. Asimismo, el análisis indica que, en congruencia con nuestra hipótesis, usar redes sociales lleva a creer más en fake news, pero contrario a lo esperado, consumir medios tradicionales también deriva en mayor creencia de noticias falsas. Particularmente, el estudio exploró el rol mediador de la creencia en fake news en el efecto del uso de medios tradicionales y sociales en el seguimiento de medidas preventivas. Se encontró evidencia de este efecto indirecto: el uso de medios tradicionales y sociales es un predictor de creer fake news sobre COVID-19, lo cual resulta en un menor acatamiento de medidas. En cambio, el efecto directo de usar medios tradicionales y sociales sobre el acatamiento de medidas tiene una dirección positiva. Así, este trabajo evidencia que las fake news pueden obstaculizar la resolución de la crisis sanitaria, desincentivando el cumplimiento de estrategias precautorias.
\end{abstract}

Palabras clave

Consumo de medios, desinformación, COVID-19, redes sociales, fake news, México.

Forma sugerida de citar: Galarza-Molina, R., \& Muñiz, C. (2021). La creencia en fake news y su rol en el acatamiento de medidas contra COVID-19 en México. Universitas-XXI, 35, pp. 19-38. https://doi.org/10.17163/uni.n35.2021.01 


\begin{abstract}
During the COVID-19 pandemic, fake news proliferated along with the concern that they would affect behavior regarding the disease. With a semi-representative survey in Mexico $(N=1211)$, this study analyzes a mediational process to determine the impact of the use of traditional and social media on compliance with contagion prevention measures, through the perception of veracity of fake news about COVID-19. As anticipated, results indicate that believing fake news leads to less compliance of preventive measures. Likewise, the analysis indicates that, consistent with our hypothesis, using social media leads to more belief in fake news, but contrary to our expectations, consuming traditional media also leads to a greater belief in fake news. In particular, the study explored the mediating role of belief in fake news on the effect that using traditional and social media has on compliance with preventive measures. We found evidence for this indirect effect: use of traditional and social media is a predictor of believing fake news about COVID-19, which then results in lower compliance with measures. In contrast, the direct effect of using traditional and social media on compliance with measures has a positive direction. Thus, this work evinces that fake news can hinder the resolution of the health crisis, by discouraging compliance with preventive strategies.
\end{abstract}

\title{
Keywords
}

Media use, COVID-19, disinformation, social media, fake news, México.

\section{Introducción}

Si bien la pandemia por la enfermedad COVID-19 no es la primera que enfrenta la humanidad, una diferencia señalada por la Organización Mundial de la Salud (OMS) respecto de otras contingencias similares es que el fenómeno presente se amplifica por las prácticas y posibilidades actuales que los seres humanos tenemos de viajar más lejos y más rápido (Zarocostas, 2020). Un problema similar se ha identificado con la información acerca del padecimiento, de tal forma que múltiple información, tanto verídica como falsa, tiene amplio alcance gracias al internet y a las redes sociales. Durante la crisis por el coronavirus esta dinámica ha generado lo que la OMS nombró como infodemia, es decir, la difusión rápida de todo tipo de información, lo cual dificulta la resolución del problema (OMS, 2020). De acuerdo con Sylvie Brand, encargada de la estrategia de la OMS para contrarrestar la infodemia, el reto ante este problema es procurar que la gente 
reciba información correcta para actuar de manera apropiada y mitigar el impacto de la enfermedad (Zarocostas, 2020).

Históricamente, los medios de información han llevado a cabo ese rol primordial en momentos críticos, por lo que el entendimiento público sobre el problema en cuestión se configura a partir de los mensajes mediáticos (Picard \& Yeo, 2011). Tanto en la presente crisis como en anteriores - la del AH1N1 por ejemplo - la población ha recurrido a los medios para aminorar la incertidumbre sobre la situación (Muñiz, 2011). No obstante, en el escenario actual en el que la gente se informa también a través de redes sociales, los medios tradicionales han perdido la hegemonía en el flujo de información que impacta en la opinión pública (Vos \& Thomas, 2018). Estas condiciones favorecen la circulación de desinformación sobre la enfermedad, desde la autenticidad de su origen, las medidas del gobierno para controlarla, las formas de contagio y las posibles curas. La principal preocupación ante esta abundancia de falsedades, es que la verdad en estas circunstancias es cuestión de vida o muerte (Pennycook et al., 2020), ya que creer en las noticias falsas puede llevar a desestimar la COVID-19, automedicarse con remedios inútiles o peligrosos y a desacatar las instrucciones de la autoridad, poniendo en riesgo la salud de la población y la habilidad de los gobiernos para ser efectivos (Pulido et al., 2020).

El tema de las fake news ha adquirido interés en los últimos años (sobre todo en lo que se refiere a desinformación política) y gradualmente se han conocido aspectos relevantes alrededor de él, como, por ejemplo, el rol de los hábitos de consumo de medios en percibir esta información como verdadera. Este trabajo pretende contribuir a esta literatura, situando el estudio de la desinformación en el contexto mexicano durante los primeros meses de la pandemia, con el objetivo de presentar evidencia sobre qué tan extendida es la creencia de información falsa sobre COVID-19 en México, así como la relación entre el uso de medios (tradicionales y sociales), la percepción de veracidad de la desinformación sobre COVID-19 y el acatamiento de medidas preventivas ante la pandemia.

\section{COVID-19 en México}

En México, el primer caso de COVID-19 se confirmó el 27 de febrero de 2020. Para el 21 de abril, el país había entrado en fase 3, ante la evidencia de brotes activos y propagación en todo el país (Suárez et al., 2020). En octubre de 2020, la cifra reportada por las autoridades federales era de alrededor de 854 mil casos y más de 86 mil muertes (Gobierno de México, s.f.). 
Paralelo a la expansión del virus, las noticias falsas sobre la enfermedad también impactaron a la población mexicana. Un recuento realizado por el Sistema Público de Radiodifusión del país identificó que entre marzo y julio de 2020 circularon 1294 noticias falsas (Zapata, 2020). En las primeras semanas de la epidemia la gran mayoría de usuarios de WhatsApp (90\%), Instagram (91\%), Twitter (89 \%), YouTube (83\%) y Facebook $(88 \%)$ detectaron una alta circulación de noticias falsas en tales plataformas (Universidad Nacional Autónoma de México, 2020).

\section{Cobertura de medios sobre salud}

Los mensajes sobre salud en medios constituyen un importante recurso y tienen una influencia notable en la sociedad contemporánea (Seale, 2002). Este contenido mediático puede tener un impacto tanto en el comportamiento individual, como en las ideas que se tienen acerca de políticas públicas a nivel colectivo (Bryant et al., 2013). Estos efectos se han detectado tanto para información publicada en medios tradicionales como para la información que se encuentra en internet, el cual en las últimas décadas ha facilitado los procesos de recolección de información sobre salud para tomar decisiones (Soederberg-Miller \& Bell, 2012). Sin embargo, la información sobre salud en línea ha sido cuestionada pues existe evidencia de que una gran proporción es información falsa (Wang et al., 2019). La popularidad de esta información falsa puede tener consecuencias perniciosas para la salud pública y decisiones de salud individuales (Pulido et al., 2020). La desinformación de salud que más abunda en línea está relacionada con el tema de vacunas y enfermedades infecciosas que han tenido importancia en años recientes como el ébola y el zika (Wang et al., 2019). Esta tendencia parece replicarse con la pandemia por la COVID-19, durante la que, ante el incipiente conocimiento sobre la enfermedad y en medio de la incertidumbre, surgieron rumores y narrativas basadas en información falsa (Xaudiera \& Cardenal, 2020).

\section{¿Qué son las fake news?}

El uso de información no verificada con fines de manipular a la opinión pública no es nuevo y puede ser rastreado incluso a la antigua Grecia (Ga- 
rrett, 2011). No obstante, las características del entorno mediático actual, en particular gracias a las redes sociales, han favorecido la diseminación de información falsa debido a los atributos de estas plataformas, que facilitan el crear y compartir contenido y conectan a usuarios en red (Al Rawi, 2019). Tales características implican menos filtros respecto a los mensajes que se difunden y un mayor potencial que estos se hagan virales. A estas diferencias respecto a medios tradicionales que solían ser el principal mecanismo para adquirir y hacer sentido de la información sobre el entorno, se suma que las redes sociales han propiciado importantes cambios en los hábitos de consumo informativo, constituyéndose cada vez más en una de las principales fuentes a través de las cuales la ciudadanía encuentra noticias (Song et al., 2020). En México, un $22 \%$ de la población utiliza las redes sociales para buscar información (Instituto Federal de Telecomunicaciones, 2019). Estas condiciones pueden exacerbar los efectos de mensajes engañosos (Garrett, 2019), porque el público pasa cada vez más tiempo en un entorno en el que la desinformación se crea y circula fácilmente (Bridgman et al., 2020).

De tal manera, el fenómeno de la desinformación ha adquirido interés no solo a nivel académico sino en la discusión pública, particularmente alrededor del concepto de las fake news. Este término tampoco es nuevo y su uso ha evolucionado, de ser empleado anteriormente para referirse a sátira política, noticias parodia y propaganda (Tandoc Jr. et al., 2019) hasta el uso que se le da en la actualidad, sobre todo tras la elección de Donald Trump a la presidencia de Estados Unidos, para hablar de fake news como un género de historias pseudo periodísticas fabricadas para desinformar (Jones-Jang et al., 2020). En uno de los primeros estudios sobre este tema en el contexto de la mencionada elección estadounidense, Allcott y Gentzkow (2017) definen las fake news como "noticias intencional y verificablemente falsas, que pueden engañar a los lectores" (p. 213). Otra característica importante de estos formatos noticiosos es que buscan asemejarse a notas periodísticas convencionales (Tandoc Jr., 2019). Las dos motivaciones que suelen asociarse con este tipo de contenido falso son: el interés económico, pues los clics atraídos hacia estas notas pueden ser monetizados mediante la venta de anuncios; y el interés ideológico o político, con el fin de desprestigiar a ciertos personajes públicos o empujar cierta agenda ideológica (Allcott \& Gentzkow, 2017). 


\section{Estudios previos sobre fake news}

Las fake news se han convertido en una fuente de preocupación para actores públicos y organizaciones civiles (Wasserman \& Madrid-Morales, 2019) y objeto de estudio de académicos por el posible impacto en la opinión pública, llevando a individuos a tomar decisiones basadas en información incorrecta (Tandoc, Jr. et al., 2019). Las investigaciones acerca de este tema se han interesado en entender diversos aspectos relacionados al fenómeno, desde cuál es su alcance (Allcott \& Gentzkow, 2017); quiénes creen y comparten este contenido (Allcot \& Gentzkow, 2017; Bridgman et al., 2020; Garrett, 2019; Calvillo et al., 2020; Guess et al., 2019; Jamieson \& Albarracín, 2020); los procesos cognitivos que intervienen para creer o no en información falsa (Bago et al., 2020); definiciones del concepto por parte de periodistas (Tandoc Jr. et al., 2019) y del público (Wagner \& Boczkowski, 2019; Nielsen \& Graves, 2017); y en menor medida, las consecuencias en las actitudes ciudadanas respecto a aspectos como confianza en medios (Van Duyn \& Collier, 2019; Wasserman \& Madrid-Morales, 2019) y cinismo político (Jones-Jang et al., 2020).

Una variable que ha sido encontrada como factor determinante en términos de quién cree y comparte las noticias falsas es la edad, aunque las conclusiones han sido contradictorias. Allcott y Gentzkow (2017) establecen que las personas de más edad creen menos en noticias falsas mientras que Guess et al. (2019) encuentran que personas mayores de 65 años comparten más este tipo de contenido. La ideología política también ha sido señalada como variable importante: personas conservadoras son más propensas a compartir información falsa (Guess et al., 2019) y a tener dificultad para distinguir entre noticias falsas y reales (Calvillo et al., 2020). Finalmente, otro factor a considerar es el nivel educativo, ya que las personas con más educación tienden a no creer en noticias falsas y a tener nociones acertadas acerca de las noticias (Bârgăoanu \& Radu, 2018; Nyhan \& Reifler, 2012).

Adicionalmente, se han encontrado algunas diferencias importantes respecto a quién cree en noticias falsas de acuerdo con sus hábitos de consumo de medios. Allcott y Gentzkow (2017) señalan que quienes consumen más información son menos propensos a creer en noticias falsas. Garrett (2019) por su parte, indica que, durante la elección presidencial estadounidense en 2012, usuarios de redes sociales tendieron a creer más en noticias falsas sobre el presidente Barack Obama, pero que, en 2016, usuarios de Facebook 
fueron menos propensos a creer en información falsa. Estas diferencias son relevantes particularmente en un contexto en el que, como se mencionó antes, cada vez más personas se informan por redes sociales.

\section{Desinformación y COVID-19}

En vista del reto que significa la presencia de desinformación para la comunicación efectiva necesaria en torno a la enfermedad COVID-19, la academia ha enfocado su atención en investigar el fenómeno en el contexto de la pandemia, que ha sido llamada como la peor ola de desinformación (Valera, 2020). Algunos de estos trabajos detectaron la alta presencia de información falsa en redes sociales (Bridgman et al., 2020; Moscadelli et al., 2020; Pulido et al., 2020; Xaudiera \& Cardenal, 2020). Por ejemplo, un análisis de contenido realizado por Bridgman et al. (2020) en mensajes publicados en Twitter y artículos noticiosos de medios canadienses encontró que la desinformación estuvo más presente en la red social que en los otros medios analizados. En otro estudio centrado en Twitter, Pulido et al. (2020) determinaron que la información falsa se tuiteaba el doble de veces que información basada en evidencia, aunque esta última obtenía más retuits. Moscadelli et al. (2020) reportaron que hasta un $23 \%$ de los enlaces compartidos en internet en Italia contenían noticias falsas.

Por otra parte, trabajos más recientes sobre desinformación durante la crisis del coronavirus han pretendido elucidar cuáles son los predictores de percibir este contenido como verdadero. Calvillo et al. (2020) establecieron que ser de ideología conservadora es un predictor para tener menor capacidad de discernir correctamente entre noticias falsas y reales sobre la enfermedad, además para sentirse personalmente menos vulnerable al virus. Otras investigaciones se han enfocado en el rol del uso de redes sociales y la percepción de veracidad de información falsa sobre COVID-19 por parte del público. Los hallazgos de Jamieson y Albarracín (2020) apuntan que quienes reportan informarse en plataformas de redes sociales, entre la población estadounidense, están más desinformadas sobre el coronavirus y creen más información falsa. De manera similar en el contexto canadiense, Bridgman et al. (2020) señalan que quienes consumen más información noticiosa en medios tradicionales tienen menos nociones erróneas sobre el padecimiento, contrario a quienes se informan vía redes sociales, que creen más en la desinfor- 
mación sobre la enfermedad COVID-19. Además, estos autores encontraron que, a su vez, creer en información falsa sobre la COVID-19 está vinculado con un menor acatamiento de las medidas de distanciamiento social.

Aunque estos hallazgos son valiosos para entender mejor este fenómeno, Wasserman y Madrid-Morales (2019) recalcan que, a pesar de tratarse de un problema detectado a nivel mundial, la mayoría de la literatura actual se ha concentrado en el Norte Global. De tal forma, este estudio pretende unirse a la línea de investigación emprendida internacionalmente pero que aún es incipiente en México, con la finalidad de contribuir al entendimiento del impacto de la desinformación en este país. Tomando en consideración los antecedentes y evidencia empírica descrita, en este artículo se plantean las siguientes hipótesis y pregunta de investigación:

H1. Percepción de veracidad de fake news estará negativamente relacionada con acatamiento de medidas de prevención de contagio.

H2. Usar redes sociales para informarse sobre COVID-19 será un predictor de creer en fake news sobre COVID-19.

H3. Usar medios tradicionales para informarse sobre COVID-19 será un predictor de no creer en fake news sobre COVID-19.

PI 1. ¿El uso de redes sociales (medios tradicionales) impactará negativamente (positivamente) el acatamiento de medidas de prevención de contagio a través del nivel de percepción de veracidad de fake news?

\section{Método}

Con el propósito de evaluar las hipótesis y pregunta planteadas, se realizó una encuesta nacional en línea, administrada por la empresa QuestionPro, utilizando un muestreo probabilístico polietápico que toma como referencia las zonas Nielsen de México. La encuesta se aplicó entre el 1 y 8 de septiembre de 2020 a 1211 personas mayores de edad.

La muestra está compuesta de la siguiente forma. En cuanto a sexo, 50,9 $\%$ se identificó como mujer y 49,1\% como hombre. Las edades oscilaron entre 18 y 79 años $(M=39 ; S D=13.83)$. En términos de nivel educativo, 9 $\%$ reportó tener estudios de secundaria o menos, $32 \%$ tenía estudios de preparatoria, 52,4\% tenía estudios universitarios y 6,6 \% tenía posgrado. El 19 $\%$ de los encuestados se encontraba en los dos niveles económicos más altos de acuerdo con la clasificación Nielsen $(\mathrm{ABC}+), 34 \%$ se encontraba en 
los niveles intermedios (C/C-) y $47 \%$ se encontraba en los dos niveles más bajos (D/D+).

El cuestionario aplicado contenía preguntas para evaluar las siguientes variables de interés para este estudio:

Uso de redes sociales. Se pidió a los encuestados que contestaran en una escala del 1 al 5 ( 1 = Nunca, 5 = Todos los días), qué tan seguido se informaron sobre la pandemia a través de: Facebook, Twitter, WhatsApp y YouTube. Se promediaron las respuestas a estas cuatro preguntas para generar un índice compuesto $(\mathrm{M}=3.12 ; \mathrm{DE}=1.01 ; \alpha=.76)$.

Uso de medios tradicionales. Los encuestados reportaron en una escala del 1 al 5 (1= Nunca, 5 = Todos los días) qué tan seguido se informaron sobre la pandemia a través de noticias o información en: periódicos impresos, en la radio, en televisión y en periódicos digitales. Las respuestas a estos cuatro ítems fueron promediadas $(\mathrm{M}=3.33 ; \mathrm{DE}=.90 ; \alpha=.72)$.

Percepción de veracidad de fake news sobre COVID-19. Se pidió a los participantes que evaluaran su nivel de acuerdo y desacuerdo ( 1 = Nada, 5 $=$ Totalmente) con siete afirmaciones que hacían referencia a rumores o información falsa que circuló respecto al COVID-19, como por ejemplo "El origen del coronavirus no es natural, sino que este fue creado por científicos en un laboratorio" y "La enfermedad COVID-19 no existe". Las respuestas fueron promediadas para generar la escala $(\mathrm{M}=2.08 ; \mathrm{DE}=.87 ; \alpha=.82)$.

Acatamiento de medidas de prevención de contagio. Los participantes reportaron en una escala del 1 al 5 ( $1=$ Nunca, $5=$ Muy a menudo) qué tanto han cumplido con una serie de medidas para prevenir contagiarse de la enfermedad. Entre las 18 preguntas, se cuestionó, por ejemplo, acerca del uso de cubrebocas para cubrir nariz y boca, evitar lugares públicos con mucha gente, trabajar desde casa, cancelar o retrasar viajes en avión, tren o autobús y lavado de manos. Las respuestas fueron promediadas $(\mathrm{M}=4.19 ; \mathrm{DE}$ $=.66 ; \alpha=.92)$.

Asimismo, se utilizaron como variables de control, variables sociodemográficas como sexo (hombre $=1$, mujer $=0$ ), edad, nivel socioeconómico $(\mathrm{ABC}+=1$, Otros $=0)$, nivel educativo (Con estudios universitarios $=1$, Sin estudios universitarios $=0$ ), ideología política - medida en una escala del 1 al $10(1=$ izquierda, $10=$ derecha $)-$ y preferencia de partido político $($ Apartidista $=1$, No apartidista $=0)$. 


\section{Resultados}

La Tabla 1 muestra las correlaciones parciales de las variables que se incluyeron en el estudio. Este análisis permite identificar si existe colinealidad entre las variables de interés, controlando los efectos de las variables sociodemográficas. La correlación más alta en esta tabla resultó entre el uso de medios tradicionales y el uso de redes sociales, rparcial $(1203)=.503, \mathrm{p}<$ .001 , por lo que se descartan problemas de colinealidad, al no existir correlaciones superiores a $r=.90$. Adicionalmente, este análisis arroja relaciones significativas entre las variables bajo estudio. La percepción de veracidad de fake news está relacionada positivamente con uso de medios tradicionales, rparcial $(1203)=.179, \mathrm{p}<.001, \mathrm{y}$ con uso de medios sociales, rparcial $(1203)=.244, \mathrm{p}<.001$. Igualmente, el acatamiento de medidas de prevención de contagio se relaciona positivamente con el uso de medios tradicionales rparcial $(1203)=.327, \mathrm{p}<.001 \mathrm{y}$ con el uso de medios sociales, rparcial $(1203)=.265, \mathrm{p}<.001$. Por otro lado, la percepción de veracidad de fake news se relaciona negativamente con el acatamiento de medidas de contagio, $\operatorname{rparcial}(1203)=-.111, \mathrm{p}<.001$.

Para evaluar las hipótesis y pregunta de investigación, se realizaron análisis mediacionales utilizando la macro PROCESS (Hayes, 2013) ejecutada en el software para procesamiento de datos SPSS. De acuerdo con las relaciones hipotetizadas, se seleccionó el modelo 4, con un bootstrapping de 5000 muestras y un nivel de confianza de $95 \%$, para evaluar el rol de creer en fake news sobre COVID-19 en el impacto del uso de medios sociales y tradicionales sobre el acatamiento de medidas de prevención de contagio.

Respecto al primer análisis, en el que se consideró el uso de redes sociales como variable independiente, la percepción de veracidad de fake news como mediadora y el acatamiento de medidas de prevención como dependiente, los resultados se explican a continuación. El modelo muestra que el uso de redes sociales predice significativamente la variable mediadora propuesta, la percepción de veracidad de fake news sobre COVID-19, $\mathrm{F}(7,1203)=16.589, \mathrm{p}<.001, \mathrm{r} 2=.088$, y la variable dependiente, acatamiento de medidas de prevención de contagio, $\mathrm{F}(8,1202)=26.619$, $\mathrm{p}$ $<.001, \mathrm{r} 2=150$. El análisis también muestra un efecto indirecto significativo entre $\mathrm{a} * \mathrm{~b}=-.029, \mathrm{DE}=.006$, pues los intervalos de límites de confianza no cruzan cero IC [-.042, -.018]. La figura 1 muestra el modelo evaluado. En este sentido, se observa un efecto indirecto del uso de redes sociales 
sobre el acatamiento de medidas de prevención a través de la percepción de la veracidad de las fake news, siendo aquellos que más usan redes sociales los que más creen en la veracidad de fake news $(\beta=.211, \mathrm{p}<.001)$, lo que derivó en un menor acatamiento de las medidas de prevención $(\beta=$ $-.139, \mathrm{p}<.001)$.

Los resultados del segundo análisis mediacional en el que la variable independiente fue el uso de medios tradicionales indican que esta variable predice significativamente la variable mediadora propuesta, creer en fake news sobre COVID-19, $\mathrm{F}(7,1203)=11.283, \mathrm{p}<.001, \mathrm{r} 2=.061$, y la variable dependiente, acatamiento de medidas de prevención de contagio, $\mathrm{F}(8,1202)=$ $33.621, \mathrm{p}<.001, \mathrm{r} 2=.182$. Igualmente, se encontró un efecto indirecto significativo $\mathrm{a} * \mathrm{~b}=-.022, \mathrm{DE}=.005$, pues los intervalos de límites de confianza no cruzan cero IC [-.033, -.013]. La figura 2 representa estos resultados. En este sentido, se observa un efecto indirecto del uso de medios tradicionales sobre el acatamiento de medidas de prevención a través de la percepción de la veracidad de las fake news $(\beta=.173, \mathrm{p}<.001)$, siendo aquellos que más usan los medios tradicionales para informarse de la pandemia quienes mayor percepción tuvieron de la veracidad de fake news, lo que redundó en un menor acatamiento de las medidas de prevención $(\beta=-.131, \mathrm{p}<.001)$.

Estos hallazgos llevan a hacer las siguientes conclusiones sobre las hipótesis planteadas. Se acepta la hipótesis 1, ambos modelos indican una relación negativa entre creer fake news sobre COVID-19 y acatar las medidas de prevención de contagio. Se acepta también la hipótesis 2 al encontrarse que el uso de redes sociales es un predictor que impacta positivamente la percepción de creer en fake news. Por otro lado, los resultados muestran una relación positiva entre el uso de medios tradicionales y creer en fake news, por lo que la hipótesis 3 se rechaza. Respecto a la pregunta de investigación, tanto el modelo con redes sociales como variable independiente, como en el que tenía a medios tradicionales como predictora, se encontró un efecto indirecto significativo, en el que el impacto del uso de estos canales de información sobre el acatamiento de medidas de prevención de contagio estaba mediado por la variable de percepción de veracidad de fake news, contribuyendo en ambos casos el seguimiento de los canales de información a reducir el acatamiento de las medidas de seguridad a través de la creencia en la veracidad de fake news. 


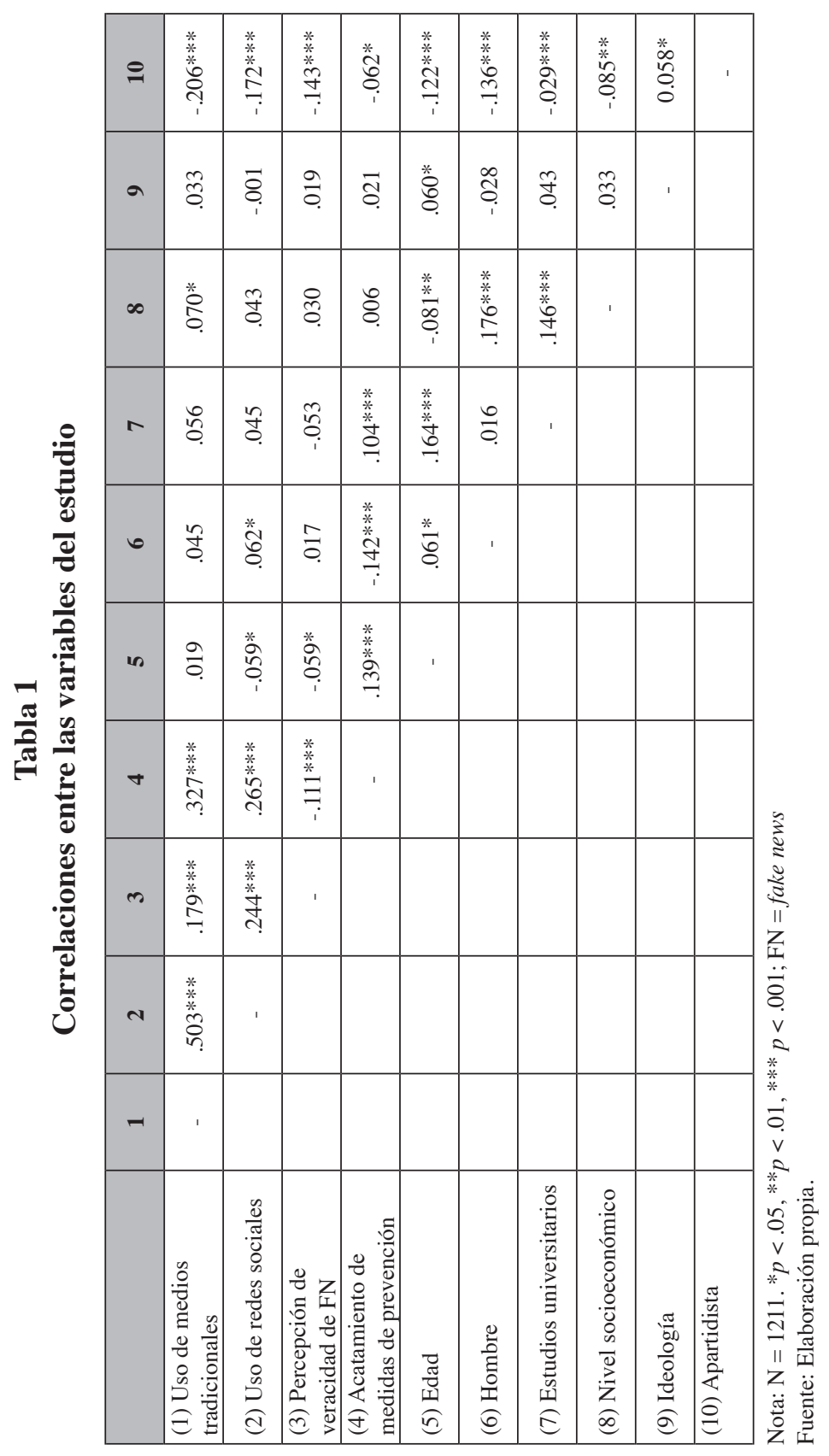




\section{Figura 1}

Modelo de mediación de percepción de Fake News en el efecto de uso de redes sociales en acatamiento de medidas de prevención

Percepción de Veracidad de Fake News
$.211^{* * * *}$

$(.024)$

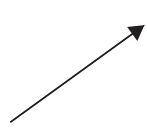

Uso de redes sociales

$$
\begin{aligned}
& \mathrm{a}^{*} \mathrm{~b}=-.029(.006), \\
& \mathrm{LLCI}=-.042 \\
& \mathrm{ULCI}=-.018
\end{aligned}
$$

Acatamiento de medidas de prevención

Nota: $* \mathrm{p}<.05 ; * * \mathrm{p}<.01 ; * * * \mathrm{p}<001 .(\mathrm{DE})$

Fuente: Elaboración propia

\section{Figura 2}

Modelo de mediación de percepción de Fake News en el efecto de uso de medios tradicionales en acatamiento de medidas de prevención

Percepción de Veracidad de Fake News

$.173^{\text {**** }}$

(.027)

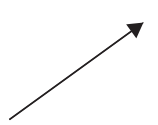

$\mathrm{a}^{*} \mathrm{~b}=-.022(.052)$,

LLCI $=-.033$,

$\mathrm{ULCI}=-.013$

Uso de medios tradicionales
$-.131^{* * *}$

(.023)

Acatamiento de medidas de prevención

Nota: * $\mathrm{p}<.05 ; * * \mathrm{p}<.01 ; * * * \mathrm{p}<001$. (DE)

Fuente: Elaboración propia 


\section{Discusión}

Este estudio buscó establecer, en el contexto de la infodemia suscitada durante la pandemia por la enfermedad COVID-19, de qué forma las noticias falsas afectan el comportamiento de la gente respecto a la enfermedad. En particular, se evaluó un proceso mediacional para determinar el impacto del uso de medios tradicionales y sociales en el acatamiento de medidas de prevención de contagio, a través de la percepción de veracidad de fake news sobre COVID-19. Con este análisis se pretende conocer más a detalle la dinámica mediante la cual, al informarse sobre la enfermedad por distintos canales en un entorno en el que la información falsa está presente, esta última puede tener consecuencias nocivas en una situación de crisis sanitaria.

Los resultados de este trabajo confirman una de las preocupaciones inmediatas respecto a los múltiples rumores y noticias falsas sobre la pandemia. Similar a lo establecido por Bridgman y otros (2020), este análisis indica que creer en fake news sobre COVID-19 conduce a un menor acatamiento de medidas de prevención de contagio como uso de cubrebocas, evitar estar en lugares públicos concurridos y guardar distancia con otras personas. Aunque destaca de manera positiva que la creencia en fake news no fue muy extendida entre la muestra encuestada, mientras que el nivel de acatamiento fue alto, la relación encontrada entre una y otra variable no deja de ser inquietante dada la naturaleza del problema - una crisis de salud - y las características de la información falsa - por referirse a remedios falsos e incluso negar la existencia del COVID-19. Bajo esas condiciones, el impacto de la desinformación, aunque sea en una pequeña porción de la población pone en riesgo la salud de esas personas y obstaculiza la contención de la epidemia.

En línea con otras investigaciones sobre fake news, un interés particular de este estudio fue establecer el impacto de los hábitos de consumo de información en la tendencia a creer en desinformación, en este caso sobre la pandemia. Trabajos anteriores han explorado el papel de las redes sociales que han sido señaladas como catalizadoras del ambiente de desinformación actual (Al Rawi, 2019). Los resultados indican que el uso de las redes sociales para obtener información acerca del COVID-19 impactan positivamente el creer en fake news, de tal manera que a mayor uso de estas plataformas (YouTube, Facebook, Twitter y WhatsApp) hay una mayor percepción de noticias falsas sobre COVID-19 como reales. Este resultado coincide con trabajos previos, que tanto para información política (Allcott \& Gentzkow, 2017, Garrett, 2019), 
como para el caso concreto del COVID-19 (Bridgman et al., 2020; Jamieson \& Albarracín, 2020), establecen este rol negativo de las redes sociales para la capacidad de discernir entre información veraz e información falsa.

Por otro lado, los resultados de este estudio respecto al rol del uso de medios tradicionales en creer en fake news fueron contrarios a la literatura anterior que indica que el utilizar este tipo de canales de información conduce a estar mejor informado y creer menos en desinformación (Allcott \& Gentzkow, 2017; Garrett, 2019), incluyendo aquella sobre COVID-19 (Bridgman et al., 2020; Jamieson \& Albarracín, 2020). En cambio, el presente análisis arrojó una relación significativa entre utilizar televisión, radio y prensa escrita y digital para informarse sobre la pandemia y el percibir noticias falsas acerca del COVID-19 como ciertas. Una posible explicación a este resultado inesperado es la saturación de información acerca del COVID-19, a través de todo tipo de canales, a seis meses de la crisis sanitaria (mientras que los estudios antes citados se hicieron al inicio de esta), lo cual pudo llevar a una acumulación de información contradictoria que ocasionó confusión sobre la enfermedad. Sin duda, la desinformación sobre COVID-19 puede tener implicaciones muy particulares, y potencialmente distintas al contenido falso sobre otros temas, por la naturaleza del problema, que ha dominado la atención y ha alterado las vidas de toda la humanidad. Asimismo, cabe destacar que la variable que se utilizó para evaluar el uso de medios se refería únicamente a la exposición a medios, sin ir a profundidad respecto a la atención a contenidos específicos, que pueden ser muy variados en cuanto a la calidad de la información que presentan. Estas diferencias pueden llegar a tener un impacto en el grado de aprendizaje acerca de un tema, lo cual a su vez podría explicar este resultado no esperado.

Por otro lado, vale la pena contemplar explicaciones alternativas que han sido esbozadas por otros autores (Von Duyn \& Collier, 2020) quienes argumentan que el discurso periodístico sobre noticias falsas conduce a mayor desconfianza en los medios y a creer en fake news. Los resultados de este estudio no permiten hacer conclusiones sobre esa posibilidad, pero conforme la desinformación se consolida como uno de los problemas más importantes del siglo XXI, el trato que dan los medios tradicionales a este tema y a este tipo de contenido falso, así como los efectos de esa cobertura, deberá explorarse más a fondo en investigaciones futuras.

Ahora bien, el análisis mediacional arroja resultados relevantes sobre la interacción entre las tres variables de interés estudiadas, que nos permite un 
mejor entendimiento de la influencia perjudicial de las fake news en el proceso de adquisición de información sobre la COVID-19 y el resultante comportamiento ante la enfermedad. Los análisis de ambos modelos arrojaron un efecto indirecto significativo entre el uso de medios tradicionales y redes sociales para informarse y seguir medidas de prevención, mediado por la creencia en fake news. De tal modo, en la cadena causal planteada en el modelo, el uso de medios y redes sociales tiene un impacto positivo en creer en notas falsas, y este efecto a su vez tiene un impacto negativo en el comportamiento para evitar el contagio. No obstante, el efecto directo entre la variable independiente (uso de medios o redes sociales) y el cumplimiento de medidas indica una relación positiva. Es decir, este análisis muestra que en la medida en la que el consumo de información a través de redes sociales y medios conduce a creer en fake news, - o sea, se presenta el efecto indirecto - cambia también la dirección de la relación entre la variable predictora y la dependiente. Si bien los resultados apuntan que los dos tipos de medios cumplen su función de influir positivamente en el público para que observen las recomendaciones estipuladas para no contagiarse (tomando en cuenta el efecto directo), las dudas que genera el mismo uso de medios y redes respecto a la veracidad de la desinformación en torno a la enfermedad, terminan por impactar el efecto en el acatamiento de manera negativa.

Así, este trabajo contribuye a la literatura acerca de fake news en México y de la desinformación sobre la pandemia por la COVID-19 a nivel mundial. Los resultados proveen evidencia del obstáculo que pueden representar las fake news para avanzar en la resolución de la crisis de salud, desincentivando el cumplimiento de medidas de prevención. Asimismo, estas conclusiones plantean interrogantes a explorar en estudios posteriores del tema.

\section{Agradecimientos y apoyos}

El presente artículo fue desarrollado en el marco del proyecto de investigación titulado "Análisis de la cobertura mediática de la pandemia de COVID-19 en México y de su impacto en el desarrollo de actitudes y comportamientos entre la ciudadanía", con clave No. 312437, aprobado por CONACYT dentro de la Convocatoria 2020-1 Apoyo para Proyectos de Investigación Científica, Desarrollo Tecnológico e Innovación en Salud ante la Contingencia por COVID-19. 


\section{Bibliografía}

Al Rawi, A. (2019). Gatekeeping fake news discourses on mainstream media versus social media. Social Science Computer Review, 37(6), 687-704. https://doi.org/10.1177/0894439318795849

Allcott, H., \& Gentzkow, M. (2017, abril). Social media and fake news in the 2016 election. (NBER Working Paper No. 23089). https://bit.ly/3sonu2Y

Bago, B., Rand, D.G., \& Pennycook, G. (2020). Fake news, fast and slow: Deliberation reduces belief in false (but not true) news headlines. Journal of Experimental Psychology: General, 149(8), 1608-1613. https://doi.org/10.1037/xge0000729

Bârgăoanu, A., \& Radu, L. (2018). Fake News or Disinformation 2.0? Some Insights into Romanians' Digital Behaviour. Romanian Journal of European Affairs, 18(1), 24-38. https://bit.ly/3tYBdiJ

Bridgman, A., Merkley, E., Loewen, P., Owen, T., Ruths, D., Teichmann, L., \& Zhilin, O. (2020). The causes and consequences of COVID-19 misperceptions: Understanding the role of news and social media. The Harvard Kennedy School Misinformation Review, 1. https://doi.org/10.37016/mr-2020-028

Bryant, J., Thompson, S., \& Finklea, B. (2013). Fundamentals of media effects. Waveland Press.

Calvillo, D.P., Ross, B.J., García, R.J.B., Smelter, T.J., \& Rutchick, A.M. (2020). Political ideology predicts perceptions of the threat of COVID-19 (and susceptibility to fake news about it). Social Psychological and Personality Science, 1-10. https://doi.org/10.1177/1948550620940539

Encuesta Nacional de Consumo de Contenidos Audiovisuales 2018 (2018). Instituto Federal de Telecomunicaciones. https://bit.ly/3n4lH2h

Garrett, K.(2011). Troubling consequences of online political rumoring.Human Communication Research, 37, 255-274. https://doi.org/10.1111/j.1468-2958. 2010.01401.x

Garrett, K. (2019). Social media's contribution to political misperceptions in U.S. Presidential elections. PLOS ONE, 14(3), 1-16. https://doi.org/10.1371/ journal.pone. 0213500

Guess, A., Nagler, J., \& Tucker, J. (2019).Less than you think: Prevalence and predictors of fake news dissemination on Facebook. Science Advances, 5. 1-8. https://doi.org/10.1126/sciadv.aau4586

Hayes, A.F. (2013). Introduction to mediation, moderation, and conditional process analysis: A regression-based approach. The Guilford Press. 
Jamieson, K., \& Albarracín, D. (2020). The relation between media consumption and misinformation at the outset of the SARS-CoV-2 pandemic in the US. The Harvard Kennedy School Misinformation Review, 1. https://doi.org/10.37016/mr-2020-012

Jones-Jang, S.M., Kim, D.H., \& Kenski, K. (2020). Predict political cynicism: Evidence from a two-wave survey during the 2018 US midterm elections. New Media \& Society, 1-21. https://doi.org/10.1177/1461444820943878

Moscadelli, A., Albora, G., Biamonte, M., Giorgetti, D., Innocenzio, M., Paoli, S., Lorini, C., Bonanni, P., \& Bonaccorsi, G. (2020). Fake News and Covid-19 in Italy: Results of a Quantitative Observational Study. International Journal of Environmental Research and Public Health, 17(5850). https://doi.org/10.3390/ijerph17165850

Muñiz, C. (2011). Búsqueda de información durante tiempos de crisis. Efectos de la comunicación interpersonal y masiva en la percepción de riesgo personal ante la gripe AH1N1. Revista de Ciencias Sociales, 17, 9-21.

Muñiz, C. (2020). Media system dependency and change in risk perception during the COVID-19 pandemic. Trípodos, 1(47), 11-26.https://bit.ly/3aJYMnG

Nielsen, K., \& Graves, L. (2017). 'News You Don't Believe': Audience Perspectives on Fake News. Reuters Institute for the Study of Journalism. https://bit.ly/3ai6RQf

Nyhan, B., \& Reifler, B. (2012). Misinformation and Fact-checking: Research Findings from Social Science. New America Foundation. https://bit.ly/3ayKmqH

Organización Mundial de la Salud (2020, abril 17). Manejo de la Infodemia: un componente clave de la respuesta mundial al COVID-19. Reporte Epidemiológico Semanal,16(95), 145-160. https://bit.ly/3geVAE6

Pennycook, G., McPhetres, J., Zhang, Y., \& Rand, D. (2020). Fighting COVID-19 misinformation on social media: Experimental evidence for a scalable accuracy nudge intervention. Psychological Science, 31(7), 770-780. https://doi.org/10.1177/0956797620939054

Picard, R., \& Yeo, M. (2011). Medical and health news and information in the UK media: The current state of knowledge. Reuters Institute for the Study of Journalism. https://bit.ly/32lbTaq

Pulido, C., Villarejo-Carballido, B., Redondo-Sama, G., \& Gómez, A. (2020). COVID-19 infodemic: More retweets for science-based information on coronavirus than for false information. International Sociology, 35(4), 377392. https://doi.org/10.1177/0268580920914755

Seale, C. (2002). Media and Health. Sage Publications. 
Soederberg-Miller, L., \& Bell, R. (2011). Online health information seeking: The influence of age, information trustworthiness, and search challenges. $J$ Aging Health, 24(3),525-41.https://doi.org/10.1177/0898264311428167.

Song, H., Gil de Zúñiga, H., \& Boomgaarden, H. (2020). Social Media News Use and Political Cynicism: Differential Pathways Through "News Finds Me" Perception. Mass Communication and Society, 23(1), 47-70. https://doi.org/10.1080/15205436.2019.1651867

Suárez, V., Suárez-Quezada, M., Oros-Ruiz, S., \& Ronquillo de Jesús, E. ( 2020). Epidemiología de COVID-19 en México: del 27 de febrero al 30 de abril de 2020. Revista Clínica Española, 220(8), 463-471. https://doi.org/ $10.1016 /$ j.rce. 2020.05 .007

Tandoc Jr., E. (2019). The facts of fake news: A research review. Sociology Compass, 13(9), 1-9. https://doi.org/10.1111/soc4.12724

Tandoc Jr., E., Jenkins, J., \& Craft, S. (2019). Fake News as a Critical Incident in Journalism. Journalism Practice, 13(6), 673-689. https://doi.org/10.108 0/17512786.2018.1562958

Universidad Nacional Autónoma de México (2020). Además de pandemia por COVID-19, México enfrenta propagación de noticias falsas. Boletín UNAM. https://bit.ly/2QwgQuc

Van Duyn, E., \& Collier, J. (2019). Priming and fake news: The effects of elite discourse on evaluations of news media. Mass Communication and Society, 22(1), 29-48. https://doi.org/10.1080/15205436.2018.1511807

Valera, S. (2020, 22 de abril). "Cristina Tardáguila: 'Estamos ante la peor ola de desinformación de la historia"”. Asociación de la Prensa de Madrid. https://bit.ly/3x4g9ZV

Vos, T., \& Thomas, R. (2018). The Discursive Construction of Journalistic Authority in a Post-Truth Age. Journalism Studies 19(13), 2001-2010. https://doi.org/10.1080/1461670X.2018.1492879

Wagner, M.C., \& Boczkowski, P. (2019). The Reception of Fake News: The Interpretations and Practices That Shape the Consumption of Perceived Misinformation. Digital Journalism, 7(7), 870-885. https://doi.org/10.1080/ 21670811.2019.1653208

Wang, Y., McKee, M., Torbica, A., \& Stuckler, D. (2019). Systematic literature review on the spread of health-related misinformation on social media. Social Science \& Medicine, 240. https://doi.org/10.1016/j.socscimed.2019.112552 
Wasserman, H., \& Madrid-Morales, D. (2019). An exploratory study of "fake news" and media trust in Kenya, Nigeria and South Africa. African Journalism Studies, 4O(1), 107-123. http://doi.org/10.1080/23743670.2019.1627230

Xaudiera, S., \& Cardenal, A. (2020). Ibuprofen narratives in five European countries during the COVID-19 pandemic. The Harvard Kennedy School Misinformation Review, 1 . https://doi.org/10.37016/mr-2020-029

Zapata, A. (2020, agosto 16). De marzo a julio, 1, 294 notass falsas sobre covid-19 en Méxicol. Excelsior. https://bit.ly/3ggTPX2

Zarocostas, J. (2020, febrero 20). How to fight an infodemic. The Lancet. https://bit.ly/3ebUVRc 